\title{
Stereotactic radiosurgery for intraventricular brain metastases
}

\author{
Clinical article
}

\author{
Benjamin Farnia, B.A., ${ }^{1}$ K. Ranh Voong, M.D., ${ }^{1}$ Paul D. Brown, M.D., ${ }^{1}$ \\ Pamela K. Allen, Ph.D., ${ }^{1}$ Nandita Guha-Thakurta, M.D., ${ }^{2}$ Sujit S. Prabhu, M.D., ${ }^{3}$ \\ Ganesh Rao, M.D., ${ }^{3}$ Qianghu Wang, Ph.D., ${ }^{4}$ Zhongxiang Zhao, Ph.D., 5 \\ and Anita Mahajan, M.D. ${ }^{1}$
}

Departments of ${ }^{1}$ Radiation Oncology, ${ }^{2}$ Diagnostic Radiology, ${ }^{3}$ Neurosurgery, ${ }^{4}$ Bioinformatics and Computational Biology, and ${ }^{5}$ Radiation Physics, The University of Texas MD Anderson Cancer Center, Houston, Texas

\begin{abstract}
Object. The authors' institution previously reported a 69\% rate of crude local control for surgical management of lateral ventricle metastases at the University of Texas MD Anderson Cancer Center. For comparison, the authors here report their institutional experience with use of stereotactic radiosurgery (SRS) to treat intraventricular metastases.

Methods. To identify patients with intraventricular metastases for this retrospective review, the authors queried an institutional SRS database containing the medical records of 1962 patients with 5800 brain metastases who consecutively underwent SRS from June 2009 through October 2013. End points assessed were local control (crude and locoregional), distant failure-free survival, progression-free survival, and overall survival.

Results. Of the 1962 records examined, those for $25(1.3 \%)$ patients with $30(0.52 \%)$ intraventricular metastases were identified. Median patient age at SRS was 55.8 years. The most common primary malignancy was renal cell carcinoma $(n=13)$, followed by melanoma $(n=7)$ and breast adenocarcinoma $(n=5)$. Median tumor volume was $0.75 \mathrm{~cm}^{3}$ (range $\left.0.01-5.6 \mathrm{~cm}^{3}\right)$. Most lesions were located in the lateral ventricles $(\mathrm{n}=25,83.3 \%)$ and were treated to a median dose of $20 \mathrm{~Gy}$ (range 14-20 Gy). A total of $12(48 \%)$ patients received whole-brain radiation therapy, most $(n=10)$ before SRS. With a median follow-up of 11.4 months (range 1.6-39.2 months), the rate of crude local control was $93.3 \%$, and the rates of 6-month and 1-year actuarial locoregional control were $85.2 \%$ and $56.2 \%$, respectively. The median overall survival time after SRS was 11.6 months (range 1.3-38.9 months), and the 6-month and 1-year actuarial rates were $87.1 \%$ and $46.7 \%$, respectively. Disease dissemination developed in $7(28 \%)$ patients as a second intraventricular metastatic lesion $(n=3,12 \%)$, leptomeningeal disease $(n=3,12 \%)$, or both $(n=1,4 \%)$. Radiographic changes developed in $5(20 \%)$ patients and included necrosis $(n=2,8 \%)$ and hemorrhage $(n=3,12 \%)$. A primary diagnosis of renal cell carcinoma was associated with an improved rate of distant failure-free survival ( $p$ $=0.05)$ and progression-free survival $(\mathrm{p}=0.08)$.

Conclusions. SRS provides excellent local control for intraventricular metastases, with acceptable treatmentrelated toxicity, thereby supporting nonsurgical treatment for these lesions. The propensity for intraventricular dissemination among intraventricular metastases seems to be histologically dependent.

(http://thejns.org/doi/abs/10.3171/2014.8.GKS141354)
\end{abstract}

$\begin{array}{llll}\text { KEY WORDS } & \bullet \quad \text { intraventricular metastases } & \bullet \quad \text { stereotactic radiosurgery } \\ \text { outcomes } & \bullet & \text { brain metastases } & \text { renal cell carcinoma }\end{array}$

I NTRAVENTRICULAR metastases are rare; the literature suggests that theses metastases develop in less than $1 \%-5 \%$ of all cancer patients. ${ }^{7,911,15,18,19}$ Although optimal treatment strategies have not been established, most patients receive local therapy in the form of either resection or stereotactic radiosurgery (SRS). As previously reported,

Abbreviations used in this paper: KPS = Karnofsky Performance Scale; $\mathrm{SRS}=$ stereotactic radiosurgery; $\mathrm{WBRT}=$ whole-brain radiation therapy. our institution (The University of Texas MD Anderson Cancer Center) has examined the role of resection in the management of lateral ventricle metastases and found a crude local control rate of $69 \%$ and an overall complication rate of $52 \% .^{7}$ Resection of intraventricular metastases is not always feasible, given their deep location within the brain parenchyma. ${ }^{7,20}$ Because of its noninvasive nature, SRS might be an alternative to resection that provides a satisfactory local control with minimal adverse effects. We report our experience with using SRS in the management of intraventricular metastases. 


\section{Methods}

\section{Patient Selection and Clinical Data}

To identify patients with primary and secondary intraventricular metastases, we queried an institutional SRS database containing the treatment records of 1962 patients with 5800 brain metastases who consecutively underwent SRS between June 2009 and October 2013. Intraventricular metastases were classified as primary if they arose within the ventricles or choroid plexus and secondary if they arose within the surrounding parenchyma but extended into the ventricular space (Fig. 1). Our analysis included both types of lesion but only those secondary metastases in which more than half $(50 \%)$ of the tumor volume protruded into the ventricular space. Two authors (N.G., A.M.) were the final arbiters with respect to study inclusion. Patients were excluded from analysis if the aforementioned criteria were not met or if they were clinically and radiographically lost to follow-up. However, patients with incomplete follow-up data (missing either clinical or radiographic data) were included in analysis. Our institutional review board approved this study.

Cancer stage was determined at the time of initial cancer diagnosis. Age, Karnofsky Performance Scale (KPS) score, graded prognostic assessment, and the statuses of primary and extracranial disease were each determined at the time of SRS. The use of whole-brain radiation therapy (WBRT), either before or after SRS, was systematically recorded for each patient. For evaluation of radiographic changes, including radionecrosis and hemorrhage, serial imaging studies, either CT or MRI, were conducted after treatment. Local recurrence was defined as the first date at which at least a $20 \%$ increase in the size of the treated lesion was noted. ${ }^{6}$ Distant failure was defined as the first date at which a new lesion was detected within the brain parenchyma. Leptomeningeal disease was defined as either presence of a positive CSF sample or imaging findings diagnostic of leptomeningeal disease. Second intraventricular metastases were defined using the same criteria as for initial metastases. Disease dissemination through the CSF was defined as the development of a second intraventricular metastastic lesion or leptomeningeal disease. To evaluate acute clinical toxicity, we reviewed

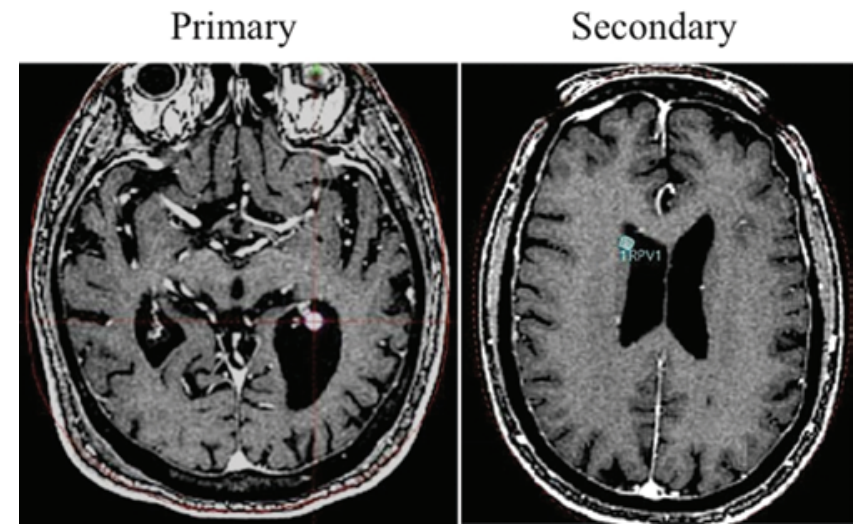

FIG. 1. A visual comparison of primary and secondary intraventricular metastases. symptoms and KPS scores obtained weekly during treatment and 1 month after treatment completion.

\section{Statistical Analyses}

To describe the cohort, we obtained frequencies and descriptive analyses of demographic and clinical characteristics. As illustrated in Table 1, when calculating descriptive statistics, 3 denominators were interchangeably used according to context: per patient $(n=25)$, per patient per treatment $(n=26)$, or per lesion $(n=30)$. In addition to local failure, we also documented locoregional failure, which included local failure as well as the development of disease dissemination through the CSF, either as a second intraventricular metastasis or leptomeningeal disease. The Kaplan-Meier method was used to calculate locoregional failure-free survival, distant failure-free survival, progression-free survival, and overall survival from the date of SRS. For calculating progression-free survival, we used the first date of local or distant brain failure as the end point. For patients who remained free of failure, the date of last imaging was used as the end point for calculations involving locoregional failure-free survival, distant failure-free survival, and progression-free survival. Patients who had leptomeningeal disease before SRS were excluded from calculation of locoregional failure-free survival. For calculations of overall survival, the date of death, or date of last follow-up evaluation if no death occurred, was used as the end point. Chi-square or Fisher exact tests were used to compare distributions of categorical variables, and Wilcoxon rank-sum tests were used to compare the distributions of continuous variables. All statistical tests were 2-sided; probability values of 0.05 or less were considered significant. All calculations were done by using Stata/MP 13.1 statistical software (StataCorp).

\section{Patient Characteristics}

Study criteria were met by $25(1.3 \%)$ patients with 30 $(0.52 \%)$ intraventricular metastases. Demographic characteristics for the cohort are shown in Table 1, and detailed patient-specific characteristics are shown in Table 2. Most patients were Caucasian $(\mathrm{n}=14,56 \%)$ and male $(\mathrm{n}=16$, $64 \%$ ). Median patient age was 55.8 years (range 27.5-85.9 years). In this cohort, radioresistant primary malignancies were overrepresented; the most common primary malignancy was renal cell carcinoma $(n=9,36 \%)$, followed by melanoma $(\mathrm{n}=7,28 \%)$ and breast adenocarcinoma $(\mathrm{n}=$ $5)$. Renal cell carcinoma accounted for the highest proportion of patients (6.8\%) with intraventricular metastases within the SRS database $(\mathrm{p}=0.0001)$. Breast adenocarcinoma $(n=5,20 \%)$, non-small cell lung cancer $(n=2,8 \%)$, esophageal adenocarcinoma $(\mathrm{n}=1,4 \%)$, and papillary thyroid carcinoma $(\mathrm{n}=1,4 \%)$ accounted for the remaining primary malignancies. Median KPS and graded prognostic assessment scores at the time of SRS were 90 (range 50-100) and 2 (range 0-4), respectively. Although disease at the primary site was either absent $(n=13,50 \%)$ or controlled $(n=9,34.6 \%)$ in most patients, extracranial disease remained uncontrolled in 15 patients $(57.7 \%)$ at the time of SRS. When compared to patients with melanoma, patients 


\section{B. Farnia et al.}

TABLE 1: Patient and tumor characteristics*

\begin{tabular}{|c|c|}
\hline Characteristic & Value $†$ \\
\hline no. of patients; no. of lesions & $25 ; 30$ \\
\hline median age; range (yrs) & $56.7 ; 27.5-85.9$ \\
\hline \multicolumn{2}{|l|}{ per patient $(n=25)$} \\
\hline \multicolumn{2}{|l|}{$\operatorname{sex}$} \\
\hline M & $16(64)$ \\
\hline $\mathrm{F}$ & $9(36)$ \\
\hline \multicolumn{2}{|l|}{ race } \\
\hline Caucasian & $14(56)$ \\
\hline Hispanic & $9(36)$ \\
\hline Asian & $1(4)$ \\
\hline black & $1(4)$ \\
\hline \multicolumn{2}{|l|}{ primary malignancy } \\
\hline renal & $9(36)$ \\
\hline melanoma & $7(28)$ \\
\hline breast & $5(20)$ \\
\hline NSCLC & $2(8)$ \\
\hline esophagus & $1(4)$ \\
\hline thyroid & $1(4)$ \\
\hline \multicolumn{2}{|l|}{ stage at initial diagnosis } \\
\hline IV & $12(48)$ \\
\hline III & $6(24)$ \\
\hline$\|$ & $5(20)$ \\
\hline 1 & $1(4)$ \\
\hline unknown & $1(4)$ \\
\hline \multicolumn{2}{|l|}{ per patient, per treatment $(n=26)$} \\
\hline median BM treated; range & $3 ; 1-11$ \\
\hline median IM treated; range & $1 ; 1-3$ \\
\hline median GPA; range & $2 ; 0-4$ \\
\hline median KPS; range & $90 ; 50-100$ \\
\hline \multicolumn{2}{|l|}{ primary site of disease } \\
\hline absent & $13(50)$ \\
\hline \multicolumn{2}{|l|}{ present } \\
\hline controlled & $9(34.6)$ \\
\hline uncontrolled & $4(15.4)$ \\
\hline \multicolumn{2}{|l|}{ extracranial disease } \\
\hline controlled & $11(42.3)$ \\
\hline uncontrolled & $15(57.7)$ \\
\hline \multicolumn{2}{|l|}{ symptoms } \\
\hline asymptomatic & $16(61.5)$ \\
\hline symptomatic & $10(38.5)$ \\
\hline headache & $6(23.1)$ \\
\hline cognitive & $4(15.4)$ \\
\hline visual & $2(7.7)$ \\
\hline ataxia & $1(3.9)$ \\
\hline \multicolumn{2}{|l|}{ per lesion $(n=30)$} \\
\hline median vol $\left(\mathrm{cm}^{3}\right)$; range & $0.75 ; 0.01-5.6$ \\
\hline median dose (Gy); range & $20 ; 14-20$ \\
\hline median prescription isodose (\%); range & $50 ; 44-89$ \\
\hline
\end{tabular}

(continued)
TABLE 1: Patient and tumor characteristics* (continued)

\begin{tabular}{cc}
\hline Characteristic & Value† \\
\hline $\begin{array}{c}\text { per lesion }(\mathrm{n}=30) \text { (continued) } \\
\text { location } \\
\text { lateral } \\
\text { posterior }\end{array}$ \\
body & $15(50)$ \\
anterior & $8(26.7)$ \\
3rd ventricle & $2(6.7)$ \\
4th ventricle & $4(13.3)$ \\
laterality & $1(3.3)$ \\
rt & \\
It & $14(46.7)$ \\
midline & $14(46.7)$ \\
origin & $2(6.7)$ \\
primary & \\
secondary & $16(53.3)$ \\
pre-SRS MRI findings & $14(46.7)$ \\
hemorrhage & \\
edema & $4(13.3)$ \\
\hline
\end{tabular}

* $\mathrm{BM}=$ brain metastasis; GPA = graded prognostic assessment; $\mathrm{IM}=$ intraventricular metastasis; NSCLC = non-small cell lung cancer.

$\dagger$ Values are number (\%) unless otherwise specified.

with renal cell carcinoma were more likely to have both uncontrolled disease at the primary site $(20 \%$ vs $0 \%$, respectively) and uncontrolled extracranial disease (70\% vs $42.9 \%$, respectively). Most patients were asymptomatic (n $=16,61.5 \%$ ), but among those who were symptomatic, the most common complaints were headache $(\mathrm{n}=6,23.1 \%)$ and cognitive disturbance $(\mathrm{n}=4,15.4 \%)$, including confusion, word-finding difficulty, and difficulty concentrating.

\section{Tumor Characteristics}

Median tumor volume was $0.75 \mathrm{~cm}^{3}$ (range 0.01-5.6 $\mathrm{cm}^{3}$ ), and lesions were most commonly primary in origin $(\mathrm{n}=16,53.3 \%)$ and located within the lateral ventricle $(\mathrm{n}=25,83.3 \%)$, specifically the posterior horn $(\mathrm{n}=15$, $50 \%)$. However, lesions within the third $(\mathrm{n}=4,13.3 \%)$ and fourth $(n=1,3.3 \%)$ ventricles were also represented. Lateral ventricle lesions were more commonly right sided $(\mathrm{n}=13,52 \%)$ and third ventricle lesions left sided $(\mathrm{n}=3$, $75 \%$ ). Median tumor size among patients with renal cell carcinoma was double that among patients with melanoma and breast adenocarcinoma: $0.76 \mathrm{~cm}^{3}$ (range $0.18-5.6$ $\mathrm{cm}^{3}$ ) versus $0.38 \mathrm{~cm}^{3}$ (range $0.01-4.2 \mathrm{~cm}^{3}$ ), respectively. With half of all primary lesions occurring in patients with renal cell carcinoma, tumor origin similarly differed based on primary malignancy. According to imaging studies conducted before SRS, most lesions were without hemorrhage $(n=26,86.6 \%)$ or edema $(n=26,86.7 \%)$.

\section{Treatment Characteristics}

Twelve (48\%) patients, each with additional parenchy- 


\section{Stereotactic radiosurgery for intraventricular brain metastases}

TABLE 2: Patient- and lesion-specific characteristics, by primary malignancy*

\begin{tabular}{|c|c|c|c|c|c|c|c|c|c|c|c|c|}
\hline $\mathrm{Pt}$ & Lesion & $\begin{array}{c}1^{\circ} \\
\text { Malignancy }\end{array}$ & $\begin{array}{c}1^{\circ} \\
\text { Lesion }\end{array}$ & Location† & WBRT $\ddagger$ & $\begin{array}{l}\text { SRS } \\
\text { Dose } \\
\text { (Gy) }\end{array}$ & Vol $\left(\mathrm{cm}^{3}\right)$ & $\begin{array}{c}\text { Time to } \\
\text { Local } \\
\text { Failure§ }\end{array}$ & $\begin{array}{c}\text { Time to } \\
\text { Distal } \\
\text { Failure§ }\end{array}$ & $\begin{array}{l}\text { Time to } \\
\text { 2nd IM§ }\end{array}$ & $\begin{array}{l}\text { Time to } \\
\text { LMD§ }\end{array}$ & $\begin{array}{l}\text { Last } \\
\text { Status }\end{array}$ \\
\hline 1 & 1 & renal & yes & posterior & - & 20 & 0.76 & - & 7 & - & - & dead \\
\hline 2 & 2 & & no & body & - & 19 & 2.80 & - & - & - & - & alive \\
\hline 3 & 3 & & yes & posterior & - & 20 & 0.61 & - & - & - & - & alive \\
\hline \multirow[t]{2}{*}{4} & 4 & & yes & posterior & - & 20 & 0.93 & - & - & - & - & dead \\
\hline & 5 & & yes & anterior & & 20 & 0.38 & - & & & & \\
\hline 5 & 6 & & yes & posterior & - & 20 & 1.90 & - & - & - & - & alive \\
\hline 6 & 7 & & no & posterior & - & 16 & 5.60 & - & - & - & - & dead \\
\hline \multirow[t]{3}{*}{7} & 8 & & yes & posterior & - & 14 & 0.27 & - & - & - & - & dead \\
\hline & 9 & & no & posterior & & 14 & 1.90 & - & & & & \\
\hline & 10 & & yes & body & & 14 & 0.19 & - & & & & \\
\hline \multirow[t]{2}{*}{$8 \pi$} & 11 & & no & fourth & - & 18 & 1.00 & - & 7.2 & 11.6 & - & dead \\
\hline & 12 & & yes & posterior & - & 20 & 0.18 & - & 1.9 & - & - & \\
\hline 9 & 13 & & no & third & - & 20 & 0.48 & 1.1 & 3.2 & - & - & dead \\
\hline 10 & 14 & melanoma & yes & posterior & before & 18 & 0.05 & - & - & - & - & dead \\
\hline 11 & 15 & & no & posterior & before & 18 & 0.87 & - & 1 & 1 & 6.6 & dead \\
\hline 12 & 16 & & yes & posterior & - & 20 & 0.93 & - & - & - & - & alive \\
\hline 13 & 17 & & yes & posterior & before & 20 & 0.01 & - & 2.4 & - & $0^{* *}$ & dead \\
\hline 14 & 18 & & yes & body & - & 20 & 0.35 & - & 2.1 & 6.6 & - & dead \\
\hline 15 & 19 & & no & body & before & 15 & 4.20 & - & 0.8 & - & 0.8 & dead \\
\hline 16 & 20 & & no & body & after & 20 & 0.07 & 2.8 & 1.4 & - & 8.7 & dead \\
\hline 17 & 21 & breast & no & third & before & 20 & 0.01 & - & - & - & - & alive \\
\hline 18 & 22 & & yes & anterior & before & 20 & 0.37 & - & - & - & - & alive \\
\hline 19 & 23 & & yes & posterior & - & 18 & 3.90 & - & 5.9 & - & - & alive \\
\hline 20 & 24 & & yes & body & before & 20 & 0.40 & - & - & - & - & alive \\
\hline 21 & 25 & & no & third & before & 18 & 0.93 & - & 2.6 & - & - & dead \\
\hline 22 & 26 & NSCLC & no & body & after & 20 & 0.29 & - & - & - & - & dead \\
\hline 23 & 27 & & no & body & before & 20 & 0.95 & - & - & - & - & dead \\
\hline \multirow[t]{2}{*}{24} & 28 & esophageal & no & third & before & 19 & 0.49 & - & 1.1 & - & - & dead \\
\hline & 29 & & no & posterior & & 19 & 0.73 & - & & & & \\
\hline 25 & 30 & thyroid & yes & posterior & - & 18 & 4.44 & - & 8.7 & 8.7 & - & alive \\
\hline
\end{tabular}

* $1^{\circ}=$ primary; $\mathrm{LMD}=$ leptomeningeal disease; $\mathrm{Pt}=$ patient; $-=$ not applicable.

$\dagger$ Anterior, body, and posterior refer to lesions within the lateral ventricles.

$\ddagger$ In relation to SRS treatment.

$\S$ Time in months, as calculated from date of SRS.

II Treated on 2 occasions for distinct lesions.

** Leptomeningeal disease developed 1.4 years before SRS.

mal metastases, received WBRT, either before $(n=10)$ or after $(n=2)$ SRS. No patient received planned sequential WBRT and SRS treatments. All patients received Gamma Knife-based SRS except 1 (Case 14), who received linear accelerator-based SRS. Lesions were treated with a median dose of $20 \mathrm{~Gy}$ (range 14-20 Gy) to the 50\% isodose line (range 44\%-89\%). Four patients (Cases 4, 7, 8, and 24) had more than 1 intraventricular lesion, and with the exception of 1 patient (Case 8), all intraventricular lesions were treated synchronously. The patient in Case 8 underwent a second course of SRS after development of a second intraventricular lesion 1 year after initial treatment. Among the 4 patients with multiple intraventricular lesions, $3(75 \%)$ had renal cell carcinoma as a primary malignancy, yielding a tumor-to-patient ratio of 1.5, second only to non-small cell lung cancer with a ratio of 2 . Of note, the patient with 3 intraventricular lesions (Case 7) had 2 periventricular lesions that were treated synchronously as well.

\section{Outcomes, Survival, and Univariate Analyses}

The follow-up records for 4 patients were incomplete: follow-up images were not available for 1 patient (Case 22), and clinical follow-up data were not available for 3 patients (Cases 12, 17, and 23). As calculated from the date of SRS, the median clinical follow-up time was 8.7 
months (range 1.2-38.9 months) and the median imaging follow-up time was 6.1 months (range 0.85-32.6 months). Among the patients with clinical follow-up data, the median KPS score 1 month after treatment was unchanged at 90 (range 50-100). Acute clinical toxicity did not develop in any patient during or after SRS. Per treatment course, $11(47.8 \%)$ patients experienced either stabilization or improvement of symptoms, but $12(52.2 \%)$ patients experienced new or progressive symptoms; for these 12 patients, 7 (58.3\%) distant brain failures developed at a median of 2.1 months (range 0.8-7.2 months) after treatment, which accounted for the symptoms.

At last follow-up evaluation, there were 2 instances of local failure, yielding a crude local control rate of 93.3\% (28 of 30). Local failures occurred in patients with renal cell carcinoma (Case 9) and melanoma (Case 16), yielding malignancy-specific crude local control rates of $92.3 \%$ and $85.7 \%$, respectively. Either a second intraventricular lesion or leptomeningeal disease developed in 6 patients (Cases 8, 11, 14-16, and 25) at a median of 7.6 months (range 1-11.6 months) and 6.6 months (range 0.8-8.7 months) after SRS, respectively, yielding a crude locoregional control rate of $72 \%$. In an additional patient with melanoma (Case 13) leptomeningeal disease had developed 1.4 years before SRS. For 5 (71.4\%) of the 7 patients with disease dissemination, including all 4 patients with leptomeningeal disease, melanoma was the primary malignancy. Crude locoregional control was worse for patients with melanoma than for patients with renal cell carcinoma: $28.6 \%$ vs $84.6 \%$, respectively $(\mathrm{p}=0.02)$. Median locoregional failure-free survival time was not reached, but 6-month and 1-year actuarial rates were $85.2 \%$ and $56.2 \%$, respectively (Fig. 2). Univariate analysis failed to correlate any patient, tumor, or treatment characteristic with locoregional failure-free survival (Table 3). Distant brain failure developed in 13 patients at a median of 2.4 months (range 0.8-8.7 months) after treatment. Among all patients, median distant failure-free survival time was 6.9 months; 6-month and 1-year actuarial rates were $56.1 \%$ and $18.4 \%$, respectively; similar results were obtained for progression-free survival calculations (Fig. 2). According to univariate analysis, renal cell carcinoma as a primary malignancy was associated with improved distant failure-free survival (HR 0.32, 95\% CI 0.10-1.03, $\mathrm{p}=0.05$ ) and progression-free survival (HR 0.35, 95\% CI $0.08-1.15, \mathrm{p}=0.08$ ). Primary, as opposed to secondary, intraventricular metastases were similarly associated with improved distant failure-free survival (HR 0.30, 95\% CI $0.10-0.94, \mathrm{p}=0.04$ ) and improved progressionfree survival (HR 0.29, 95\% CI 0.09-0.91, $\mathrm{p}=0.03$ ). Lateral ventricle lesions were associated with improved progression-free survival (HR 0.36, 95\% CI 0.11-1.19, p $=0.09$ ), whereas WBRT before SRS was associated with worse distant failure-free survival (HR 2.81, 95\% CI $0.88-8.95, \mathrm{p}=0.08$ ).

Among the 25 patients in our cohort, 9 (36\%) were alive at last follow-up evaluation; survival rates differed according to primary malignancy: $80 \%$ (4/5) for breast adenocarcinoma, 33.3\% (3/9) for renal cell carcinoma, and $14.3 \%(1 / 7)$ for melanoma. Median overall survival time was 11.6 months; 6-month and 1-year actuarial rates were $87.1 \%$ and $46.7 \%$, respectively. Univariate analysis demonstrated that older age ( $\geq 60$ years) was associated with improved overall survival (HR 0.24, 95\% CI $0.07-0.79, \mathrm{p}=0.02)$, but that WBRT prior to SRS was associated with worse overall survival (HR 3.5, 95\% CI $1.09-11.3, \mathrm{p}=0.04)$. Analysis also showed a trend toward improved overall survival for lesions larger than $1 \mathrm{~cm}^{3}$ (HR $0.32,95 \%$ CI $0.09-1.16, \mathrm{p}=0.08$ ) but a trend toward worse overall survival for patients who had intraventricular lesions with hemorrhage or edema before to SRS (HR $3.29,95 \%$ CI $0.96-11.3, \mathrm{p}=0.06$ ).

\section{Radiographic Changes}

Radiographic changes developed in 5 (20\%) patients (3 with renal cell carcinoma as a primary malignancy). These changes included necrosis (Cases 9 and 25) and hemorrhage (Cases 2, 6, and 16) at medians of 4.8 months (range 1.1-8.5 months) and 2 months (range 0.07-5.5 months), respectively. Only 1 patient (Case 2) was symptomatic; this patient visited the emergency department 2 days after SRS, and CT imaging demonstrated new hemorrhage products that had migrated from the body of the lateral ventricle (the treatment site) to the third ventricle. Of the aforementioned patients who experienced hemorrhage, 1 (Case 16) had experienced hemorrhage before SRS.

\section{Discussion}

Although patients with intraventricular metastases often receive local treatment in the form of resection or SRS, not much is known about the effectiveness of the latter treatment option. To our knowledge, this retrospective review is the first case series to examine the role of SRS in the management of patients with these lesions. Our series demonstrates that SRS provides reasonable local control for intraventricular metastases and has a low side-effect profile. In addition, we observed several histologically dependent characteristics that probably influenced prognosis. Herein we explore these findings to not only better characterize our understanding of intraventricular lesions but also to establish initial treatment recommendations.

\section{Treatment Selection: Surgery, SRS, and WBRT}

Our institution has previously examined the role of resection in patients with lateral ventricle lesions and found a crude local control rate of $69 \%$ and an overall complication rate of $52 \%$ at a median follow-up time of 6 months. $^{7}$ When the patient who had leptomeningeal disease before resection was excluded from analysis, the median overall survival time was 11.7 months. Univariate analysis identified 4 factors significantly associated with survival (number of brain metastases, renal cell carcinoma as primary malignancy, preoperative KPS score, and resection method), yet only the latter 3 remained significant on multivariate analysis.

Similar to patients in our institution's study of resection, in the SRS study reported here, most patients were male, had renal cell carcinoma as a primary malignancy, and had a singular, primary intraventricular lesion located 
Locoregional Failure Free Survival

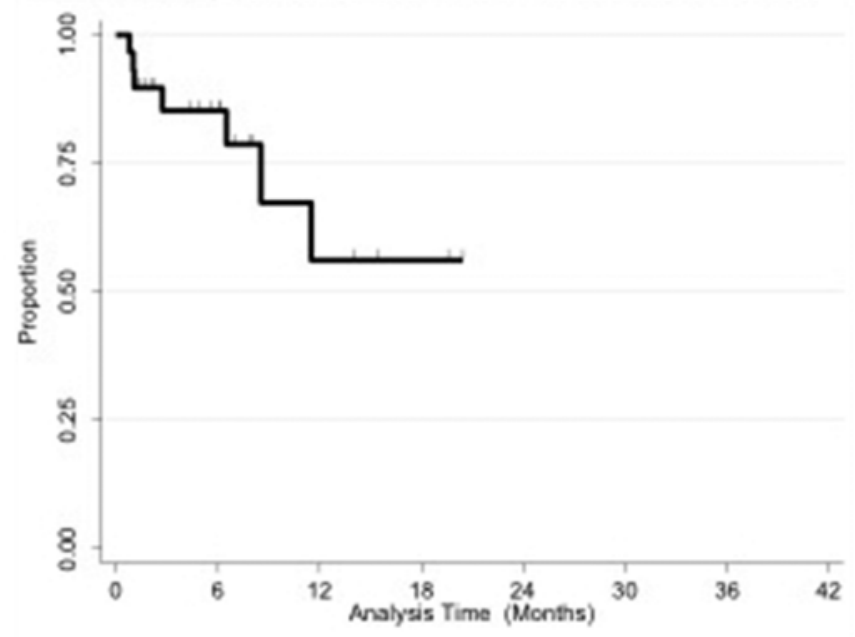

Progression Free Survival

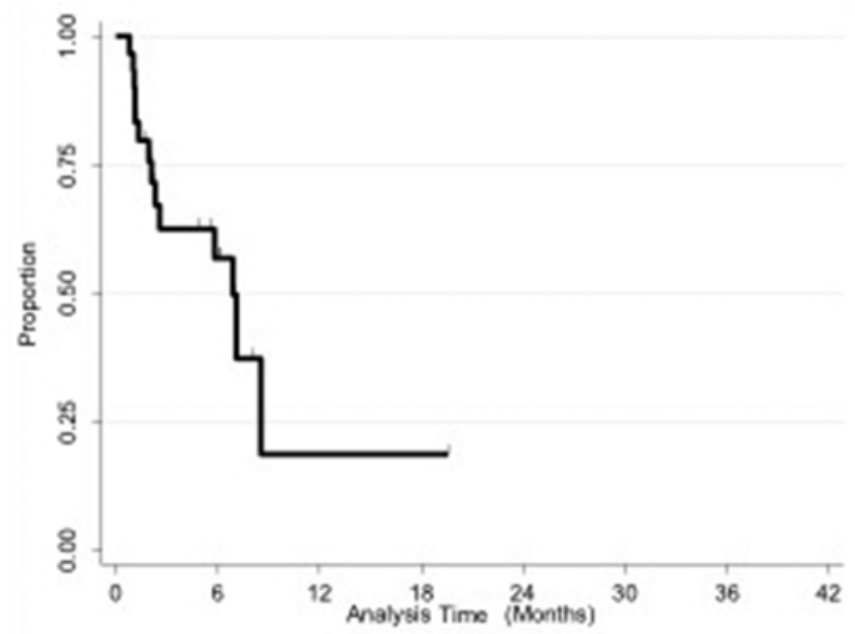

FIG. 2. Kaplan-Meier curves demonstrating survival after SRS.

in the posterior horn of the lateral ventricle. However, crude local control and treatment-related toxicity were both significantly improved in the analysis reported here: $93.3 \%$ vs $69.0 \%$ and $20.0 \%$ vs $52.0 \%$, respectively. Similar median overall survival was observed, but the comparison might not be accurate, given the longer clinical follow-up time for patients in our cohort (8.7 months vs 6 months) and the patient selection in the surgical series (including exclusion of a patient with prior leptomeningeal disease) from overall survival calculations. The results of univariate analysis also differed between the 2 studies; in our analysis of SRS, each of the aforementioned factors failed to correlate with overall survival. A variety of differences could account for these findings: for patients in the surgical series, the median KPS scores were lower (80 vs 90) and patients were more often symptomatic ( $76 \%$ vs $38.5 \%$ ), whereas patients in our SRS cohort were more likely to have uncontrolled extracranial disease $(57.7 \%$ vs $31 \%)$. Despite these differences, better outcomes were observed for patients in our SRS cohort than in our institutional surgical series.

Delivered as a minimally invasive outpatient proce-

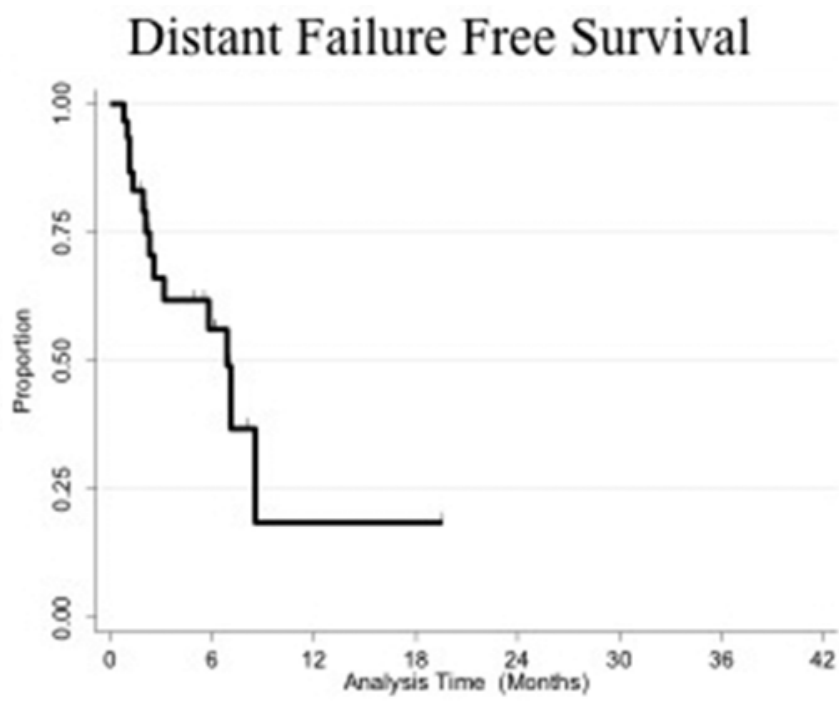

Overall Survival

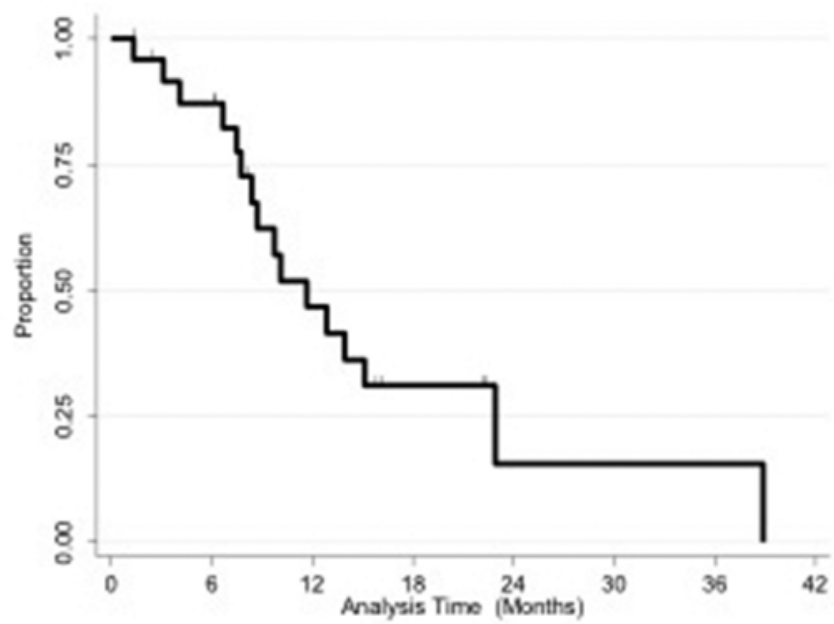

dure with very low rates of complications and death, SRS may be the preferred treatment option for select patients with intraventricular metastases, given the improved local control and fewer complications seen in our analysis. ${ }^{14}$ Additionally, SRS might better control microscopic disease left behind after incomplete resection, which is a reality in the management of intraventricular lesions, given their deep location within the brain parenchyma. ${ }^{1}$ Nevertheless, resection will probably remain the treatment of choice for patients with significant mass effect, ventricular obstruction, or severe neurological deficit. ${ }^{1,8}$

Although WBRT was not delivered in a planned sequence with SRS for any patient in our cohort, our discussion of treatment selection would be incomplete without an examination of its role as used in $48 \%$ of the patients our cohort. Rates of locoregional and distal recurrence did not statistically differ between patients treated with WBRT and those treated with SRS alone. In addition, use of WBRT before SRS failed to correlate with locoregional failure-free survival $(p=0.92)$. Median time to distal recurrence after SRS, however, was 4 times shorter for 
B. Farnia et al.

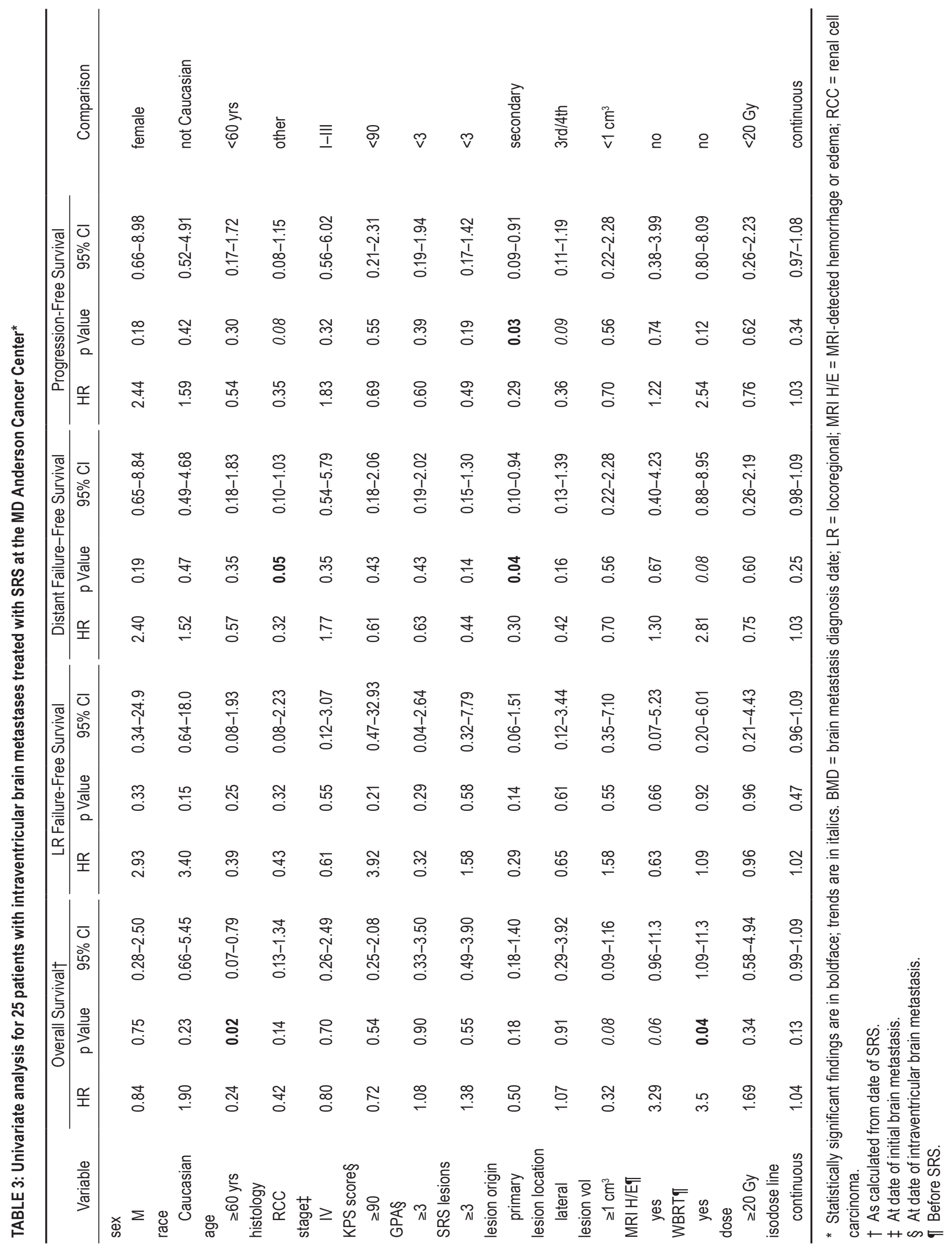




\section{Stereotactic radiosurgery for intraventricular brain metastases}

patients previously treated with WBRT (1.1 months vs 4.5 months, $p=0.04)$. Similarly, WBRT before SRS was associated with worse overall survival $(p=0.04)$ and worse distant failure-free survival $(\mathrm{p}=0.08)$, probably because of prior advanced disease. The poor outcomes for patients who had previously received WBRT might also be influenced by the primary malignancy; half of the WBRT cohort had melanoma or esophageal adenocarcinoma, both of which are associated with poor outcomes for patients who have brain metastases. ${ }^{2,3}$ Additionally, most (83.3\%) patients received WBRT before SRS, which might have allowed for the growth and selection of a radioresistant colony, considering the prevalence $(64 \%)$ of radioresistant primary malignancies in our cohort. Although the addition of WBRT will probably vary on a case-by-case basis, its use for intraventricular metastases is not likely to be of much benefit, given a combination similar locoregional and distant recurrence rates, a preponderance of patients with radioresistant primary malignancies, and the added risk for increased likelihood of cognitive decline. ${ }^{4}$

\section{Histology of Primary Malignancy}

Despite an estimated incidence of $4 \%-11 \%$, brain metastases from renal cell carcinoma accounted for nearly half (43.3\%) of all lesions among patients in our cohort. . $^{5,10,14,16}$ Given the preponderance of patients with renal cell carcinoma, it has been suggested that this tumor type probably has a tropism toward the ventricular space.,15 Although this suggestion remains to be definitively established, the implications significantly alter a patient's survival time and amount of toxicity experienced. In our study, for patients with renal cell carcinoma, rates of distant failurefree survival were improved $(p=0.05)$ and a trend toward improved progression-free survival was found $(\mathrm{p}=0.08)$ when compared with patients with other primary malignancies. This finding is particularly striking when compared with findings for patients with melanoma, the second most common primary malignancy in our cohort, because patients with renal cell carcinoma were more likely to have both an uncontrolled disease at the primary site (20\% vs $0 \%$ ) and uncontrolled extracranial disease (70\% vs $42.9 \%)$. These findings corroborate those of several recent studies that have shown that among radioresistant brain metastases, renal cell carcinoma is associated with improved survival and outcomes. ${ }^{3,12,13,17}$ We believe that this finding is probably because of a combination of differences in tumor biology among radioresistant malignancies and a variety of new chemotherapeutic agents for patients with renal cell carcinoma. Although crude local control was similar for renal cell carcinoma and melanoma, locoregional control was significantly worse for the latter: $28.6 \%$ vs $84.6 \%$, p $=0.02$. This finding suggests that the risk for disease dissemination in patients with intraventricular metastases is histologically dependent, confirming that differences in tumor biology are likely.

Many of the findings observed on univariate analysis are probably secondary to differences among primary malignancies. Because half of all primary lesions developed in patients with renal cell carcinoma, the improved rates of distant failure-free survival and progression-free survival were probably secondary to renal cell carcinoma histology. Similarly, the trend toward improved overall survival for patients with metastases greater than $1 \mathrm{~cm}^{3}$ is probably secondary to larger lesions occurring in patients with renal cell carcinoma (median, $0.76 \mathrm{~cm}^{3}$ ) compared with smaller lesions in patients with melanoma and breast adenocarcinoma (median, $0.38 \mathrm{~cm}^{3}$ ); the tendency for patients with renal cell carcinoma to have larger lesions is probably because patients with renal cell carcinoma may not undergo brain MR imaging as frequently as patients with other primary malignancies, such as melanoma. Likewise, age greater than 60 years was associated with improved overall survival probably because most $(60 \%)$ older patients had renal cell carcinoma. Last, lateral ventricle lesions were probably associated with improved progression-free survival $(\mathrm{p}=0.09)$ because renal cell carcinomas accounted for nearly half of all lateral ventricle lesions (44\%). Of course, many factors affect these findings, but with a small cohort, multivariate analysis to test whether many of these findings were truly secondary to primary histology was not feasible.

Given the magnitude of differences in primary tumor histology, we should entertain the idea of altering the SRS dose based on primary malignancy. With melanoma accounting for $71.4 \%$ of patients with disease dissemination, increasing the SRS dose for these patients might prevent spread. Central to this idea is the thought that the surrounding CSF might serve as a buffer, enabling delivery of higher doses of radiation without incurring excess damage to the surrounding parenchyma. Similarly, with radiographic changes (including radionecrosis and hemorrhage) developing in $60 \%$ of patients with renal cell carcinoma, perhaps decreasing the dose for these patients is appropriate, especially considering the improved outcomes associated with this primary malignancy. Future trials are needed to assess whether such changes in SRS dose will provide clinical benefit to patients.

Our study has several limitations, including its retrospective nature, a small, histologically diverse cohort, and limited follow-up data for some cases. Despite reporting on the second largest cohort of patients with intraventricular lesions, second only to our institutional surgical series, the size of our SRS cohort prevented the calculation of additional statistical analyses that would probably clarify our findings. As a tertiary care facility, selection bias is probably an influencing factor as well, because many patients received treatment at our center but were followed up at a local facility for which details were lacking.

\section{Conclusions}

SRS for intraventricular metastases is associated with a high rate of local control and acceptable toxicity and represents an acceptable alternative to resection. There appears to be a histologically dependent risk for dissemination of intraventricular metastases. Given the rarity of these metastases, collaborative multiinstitutional data would help validate these findings.

\section{Disclosure}

This study was supported by the National Institutes of Health/ 
National Cancer Institute, award no. P30CA016672. The authors report no conflict of interest concerning the materials or methods used in this study or the findings specified in this paper.

Author contributions to the study and manuscript preparation include the following. Conception and design: Mahajan, Farnia, Brown. Acquisition of data: Farnia, Voong, Guha-Thakurta, Wang. Analysis and interpretation of data: Mahajan, Farnia, Brown, Allen. Drafting the article: Farnia. Critically revising the article: all authors. Reviewed submitted version of manuscript: all authors. Approved the final version of the manuscript on behalf of all authors: Mahajan. Statistical analysis: Farnia, Allen. Administrative/technical/material support: Farnia, Brown. Study supervision: Mahajan, Farnia, Brown.

\section{References}

1. Barker FG II: Surgical and radiosurgical management of brain metastases. Surg Clin North Am 85:329-345, 2005

2. Bowden G, Kano H, Tempel ZJ, Caparosa E, Monaco E III, Niranjan A, et al: Gamma knife radiosurgery for management of cerebral metastases from esophageal carcinoma. J Neurooncol 118: 141-146, 2014

3. Chang EL, Selek U, Hassenbusch SJ III, Maor MH, Allen PK, Mahajan A, et al: Outcome variation among "radioresistant" brain metastases treated with stereotactic radiosurgery. Neurosurgery 56:936-945, 2005

4. Chang EL, Wefel JS, Hess KR, Allen PK, Lang FF, Kornguth DG, et al: Neurocognition in patients with brain metastases treated with radiosurgery or radiosurgery plus whole-brain irradiation: a randomised controlled trial. Lancet Oncol 10: 1037-1044, 2009

5. Culine S, Bekradda M, Kramar A, Rey A, Escudier B, Droz JP: Prognostic factors for survival in patients with brain metastases from renal cell carcinoma. Cancer 83:2548-2553, 1998

6. Eisenhauer EA, Therasse P, Bogaerts J, Schwartz LH, Sargent $\mathrm{D}$, Ford R, et al: New response evaluation criteria in solid tumours: revised RECIST guideline (version 1.1). Eur J Cancer 45:228-247, 2009

7. Hassaneen W, Suki D, Salaskar AL, Wildrick DM, Lang FF, Fuller GN, et al: Surgical management of lateral-ventricle metastases: report of 29 cases in a single-institution experience. Clinical article. J Neurosurg 112:1046-1055, 2010

8. Hazard LJ, Jensen RL, Shrieve DC: Role of stereotactic radiosurgery in the treatment of brain metastases. Am J Clin Oncol 28:403-410, 2005

9. Healy JF, Rosenkrantz H: Intraventricular metastases demonstrated by cranial computed tomography. Radiology 136:124, 1980

10. Kim YH, Kim JW, Chung HT, Paek SH, Kim DG, Jung HW: Brain metastasis from renal cell carcinoma. Prog Neurol Surg 25:163-175, 2012
11. Kohno M, Matsutani M, Sasaki T, Takakura K: Solitary metastasis to the choroid plexus of the lateral ventricle. Report of three cases and a review of the literature. J Neurooncol 27: 47-52, 1996

12. Lo SS, Clarke JW, Grecula JC, McGregor JM, Mayr NA, Cavaliere R, et al: Stereotactic radiosurgery alone for patients with 1-4 radioresistant brain metastases. Med Oncol 28 (Suppl 1): S439-S444, 2011

13. Lwu S, Goetz P, Monsalves E, Aryaee M, Ebinu J, Laperriere $\mathrm{N}$, et al: Stereotactic radiosurgery for the treatment of melanoma and renal cell carcinoma brain metastases. Oncol Rep 29:407-412, 2013

14. Maor MH, Frias AE, Oswald MJ: Palliative radiotherapy for brain metastases in renal carcinoma. Cancer 62:1912-1917, 1988

15. Matsumura H, Yoshimine T, Yamamoto S, Maruno M, Hayakawa T, Ono Y, et al: Single solitary metastasis of the slowly progressive type of renal cell carcinoma to the choroid plexus-case report. Neurol Med Chir (Tokyo) 37:916-919, 1997

16. Muacevic A, Siebels M, Tonn JC, Wowra B: Treatment of brain metastases in renal cell carcinoma: radiotherapy, radiosurgery, or surgery? World J Urol 23:180-184, 2005

17. Powell JW, Chung CT, Shah HR, Canute GW, Hodge CJ, Bassano DA, et al: Gamma Knife surgery in the management of radioresistant brain metastases in high-risk patients with melanoma, renal cell carcinoma, and sarcoma. J Neurosurg 109 Suppl:122-128, 2008

18. Raila FA, Bottoms WT Jr, Fratkin JD: Solitary choroid plexus metastasis from a renal cell carcinoma. South Med J 91: 1159-1162, 1998

19. Schreiber D, Bernstein K, Schneider J: [Metastases of the central nervous system: a prospective study. 3rd Communication: metastases in the pituitary gland, pineal gland, and choroid plexus (author's transl).] Zentralbl Allg Pathol 126:64-73, 1982 (Ger)

20. Vecil GG, Lang FF: Surgical resection of metastatic intraventricular tumors. Neurosurg Clin N Am 14:593-606, 2003

Manuscript submitted June 17, 2014.

Accepted August 6, 2014

Portions of this work were presented as a plenary oral presentation at the 17th International Leksell Gamma Knife Society Meeting, New York, New York, May 11-15, 2014.

Please include this information when citing this paper: DOI: 10.3171/2014.8.GKS141354.

Address correspondence to: Anita Mahajan, M.D., Department of Radiation Oncology, The University of Texas MD Anderson Cancer Center, 1515 Holcombe Blvd., Box 97, Houston, TX 77030. email: amahajan@mdanderson.org. 